\title{
MARVIN: Identifying Design Requirements for an AI powered Conversational User Interface for Extraterrestrial Space Habitats
}

\author{
Youssef Nahas ${ }^{*}$, Christiane Heinicke ${ }^{*}$, \\ and Johannes Schöning \\ *University of Bremen, Germany \\ ${ }^{\dagger}$ Center of Applied Space Technology and Microgravity (ZARM), Germany \\ schoening@uni-bremen.de,christiane.heinicke@uni-bremen.de
}

\begin{abstract}
In this workshop paper we report on our early work to design a conversational interface for astronaut scientists in an extraterrestrial habitat (e.g. a habitat on Moon or Mars). At the workshop we will report on our initial design and first evaluations of our conversational user interface called MARVIN. Our goal with MARVIN is to support scientists on their missions and during their daily (scientific) routines within and outside the habitat. We are installing our interface in MaMBA. The MaMBA project aims to build a first functional extraterrestrial habitat prototype.
\end{abstract}

\section{Keywords}

Conversational Interfaces - Design Requirements - Extraterrestrial Space Habitats $\cdot$ Mars $\cdot$ Moon $\cdot$ ZARM $\cdot$ NASA $\cdot$ MARVIN

How to cite this book chapter:

Nahas, Y., Heinicke, C. and Schöning, J. 2020. MARVIN: Identifying Design Requirements for an AI powered Conversational User Interface for Extraterrestrial Space Habitats. In: Loizides, F., Winckler, M., Chatterjee, U., Abdelnour-Nocera, J. and Parmaxi, A. (eds.) Human Computer Interaction and Emerging Technologies: Adjunct Proceedings from the INTERACT 2019 Workshops. Pp. 227-230. Cardiff: Cardiff University Press. DOI: https://doi.org/10.18573/book3.ae. License: CC-BY 4.0. 


\section{Motivation \& Related Work}

Today, conversational interfaces such as Google Assistant, Amazon Alexa or Siri are one of the most ubiquitous artificial intelligent (AI) systems that have been incorporated into our everyday lives. While having a long history [5-6], conversational user interfaces (CUI) just recently became more and more ubiquitous with the advent of devices like Google Home, Amazon Echo or the HomePod in our living rooms [2]. It was also shown in the past, that interaction with these devices is not trivial [8] and general design guidelines for human-AI interaction are needed [1].

While there is first work on understanding the ways people interact with conversational interfaces in everyday scenarios $[2,6,7-9]$, there is little research on how this class of AI powered interfaces can support humans in other (more professional) contexts.

Extraterrestrial habitats are an extreme example for such a professional context. It is very likely that conversational interfaces will support astronauts on their often very long and challenging missions and assist them performing research tasks during space flight or planetary exploration missions. For example, a first prototype of a CUI called CIMON ${ }^{1}$ was recently tested on the International Space Station (ISS). CIMON is a CUI that runs IBM's Watson technology to mainly help astronauts with their daily tasks (many of them not mission critical by design). Besides that, there are also many examples in Sci-Fi literature and movies. Most prominently are HAL 9000 in the movie Space Odyssey and the "Computer" in the Star Trek franchise. Nevertheless, the requirements of a CUI in a habitat on a planetary body other than Earth will be different from one in a space station orbiting Earth.

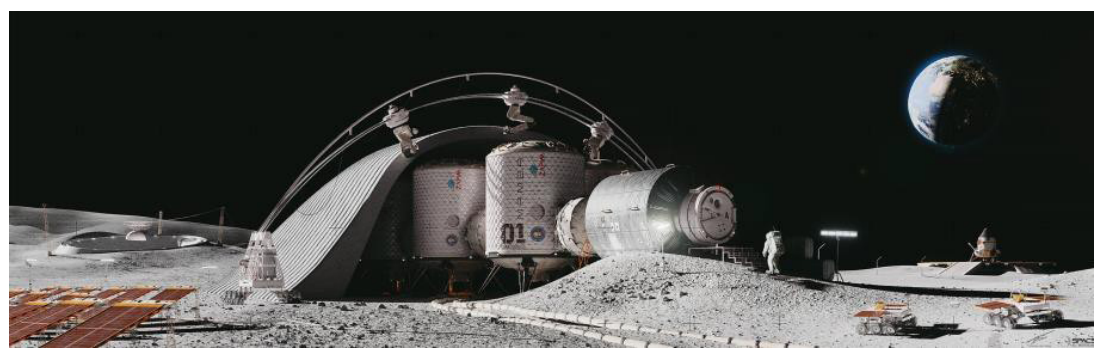

Fig. 1: Rendering of six MaMBA modules and an airlock on the Moon covered artificial cave.

${ }^{1}$ https://www.space.com/42574-ai-robot-cimon-space-station-experiment .html. 


\section{MARVIN}

We have the unique opportunity to study requirements of a CUI for a space habitat within MaMBA (Moon and Mars Base Analog). MaMBA [4] aims to build a first functional extraterrestrial habitat prototype. The habitat is developed at the ZARM in Bremen, Germany, and comprises five to six connected, but independent modules. In its final state, the habitat is intended to serve for testing technologies such as life support, power systems, and remotely operated set-ups communication [4]. A mock-up of the first module, the laboratory module, has recently been completed and first preliminary tests were run in late June 2019.

The first test consisted of a crew of 4 scientists performing biological and geological experiments in the module. During the test, we had a microphone and speaker within a 3D printed case in the module that acted as our Wizard of $\mathrm{O} z$ [3] prototype of MARVIN. The analog astronauts were instructed that there would be a CUI installed in the module, but they were not aware that MARVIN was a Wizard of $\mathrm{O} z$ prototype during the evaluation. The astronauts could trigger MARVIN and subsequently received responses from the operator. In the workshop we will report first insights on the needs and requirements of MARVIN and discuss potential challenges.

\section{References}

1. Saleema Amershi, Dan Weld, Mihaela Vorvoreanu, Adam Fourney, Besmira Nushi, Penny Collisson, Jina Suh, Shamsi Iqbal, Paul N. Bennett, Kori Inkpen, Jaime Teevan, Ruth Kikin-Gil, and Eric Horvitz. 2019. Guidelines for Human-AI Interaction. In Proceedings of the 2019 CHI Conference on Human Factors in Computing Systems (CHI '19). ACM, New York, NY, USA, Paper 3, 13 pages. DOI: https://doi.org/10.1145/3290605.3300233

2. Beirl, D., \& Rogers, Y. Using Voice Assistant Skills In Family Life. In Proceedings of Proceedings of CSCL'19, May 2019.

3. Dahlbäck, N., Jönsson, A., \& Ahrenberg, L. (1993). Wizard of Oz studieswhy and how. Knowledge-based systems, 6(4), 258-266.

4. Heinicke, C., Orzechowski, L., Abdullah, R., von Einem, M., \& Arnhof, M. (2018, September). Updated Design Concepts of the Moon and Mars Base Analog (MaMBA). In European Planetary Science Congress (Vol. 12).

5. Horvitz, E. (1999, May). Principles of mixed-initiative user interfaces. In Proceedings of the SIGCHI conference on Human Factors in Computing Systems (pp. 159-166). ACM.

6. Klopfenstein, L. C., Delpriori, S., Malatini, S., \& Bogliolo, A. (2017). The rise of bots: A survey of conversational interfaces, patterns, and paradigms. In Proceedings of the 2017 Conference on Designing Interactive Systems (pp. 555-565). ACM. 
7. Porcheron, M., Fischer, J. E., Reeves, S., \& Sharples, S. (2018, April). Voice interfaces in everyday life. In proceedings of the $2018 \mathrm{CHI}$ conference on human factors in computing systems (p. 640). ACM.

8. Dounia Lahoual and Myriam Frejus. 2019. When Users Assist the Voice Assistants: From Supervision to Failure Resolution. In Extended Abstracts of the 2019 CHI Conference on Human Factors in Computing Systems (CHI EA '19). ACM, New York, NY, USA, Paper CS08, 8 pages. DOI: https://doi.org/10.1145/3290607.3299053

9. Sciuto, A., Saini, A., Forlizzi, J., \& Hong, J. I. (2018, June). Hey Alexa, What's Up?: A Mixed-Methods Studies of In-Home Conversational Agent Usage. In Proceedings of the 2018 on Designing Interactive Systems Conference 2018 (pp. 857-868). ACM. 